\title{
Symmetric linear systems: an application of algebraic systems theory $\dagger$
}

\author{
M. HAZEWINKEL $\ddagger$ and C. MARTIN $§ \|$
}

\begin{abstract}
Dynamical systems which contain several identical subsystems occur in a variety of applications ranging from command and control systems and discretization of partial differential equations, to the stability augmentation of pairs of helicopters lifting a large mass. Linear models for such systems display certain obvious symmetries. In this paper, we discuss how these symmetries can be incorporated into a mathematical model that utilizes the modern theory of algebraic systems. Such systems are inherently related to the representation theory of algebras over fields. We will show that any control scheme which respects the dynamical structure either implicitly or explicitly uses the underlying algebra.
\end{abstract}

\section{Introduction}

Linear models of dynamical controlled systems have always been the central tool of the control engineer, and yet linear control theory has received more criticism than almost any other area of control theory. The criticisms include the point that nature is rarely linear to the point that the usual design procedures for feedback gains often completely obliterate the original dynamics of the system. The philosopher and engineer, G. Allen Smith (1981) has complained that control theorists think they could make a rock soar like an eagle if there was just a way to implement large enough gains. It is partially toward this criticism that this paper is addressed.

Often, dynamical systems are composed of several independently controlled systems interacting through some fixed structure. The structure, physical or informational in nature, is often beyond the influence of the individual controllers, or may be modified in only specific ways. To attempt to implement control procedures that violate the constraints is not acceptable. In this paper, we consider three examples of such systems and given suitable linear models. The three examples are taken from the diverse areas of aeronautics, command and control systems and numerical analysis and are only representation examples.

The example from aeronautics is the problem of stability augmentation of a pair of helicopters lifting a mass that is beyond the performance capabilities of a single helicopter. The model displays dynamical symmetries that must be respected and there are design constraints imposed by pilot workload considerations. The command and control model is loosely adapted from

Received 3 November 1982.

† This work was partially done while the second author was in residence at Erasmus University, Rotterdam.

$\ddagger$ Department of Mathematics, Erasmus University, Rotterdam, P.O. Box 1738, 3000 D R, Rotterdam, The Netherlands.

$\S$ Department of Mathematics, Case Western Reserve University, Cleveland, Ohio 44106, U.S.A.

II Supported in part by NASA Grants 2384 and DAG2-82 and DOE Contract DE-AC01-80RA-5256. 
one level of a heirarchical model proposed in various presentations at the special sessions on $C^{3}$ systems at the 1980 Conference on Decision and Control. The main features are that there are several control units representing units in a fleet action that are sharing information through fixed communication channels. There are limitations to the number of channels because of transfer of information rates and security requirements. Again control decisions must respect the underlying information structure and the inherent limitations of human command teams to assimilate information and to react with rational command decisions.

The third example comes from the very complete paper of Brockett and Willems (1974) on the discretization of partial differential equations. Their discretization procedure results in a linear system in which the state matrix is a block cyclic matrix and the control matrix has a compatible block structure. Any feedback must preserve the cyclic structure of the state matrix if the resulting system is to represent the discretization of a partial differential equation.

These three models have the shared feature that there is an underlying structure that is inherent to the system. In this paper, we develop tools from algebraic systems theory that can be used in the analysis and design of such systems. In Martin (1982), the concept of systems with symmetries is developed from an extrinsic viewpoint. In Hazewinkel and Martin (1983), the concepts are extended and the mathematics is developed from an intrinsic viewpoint. The basic idea is that the concept of symmetry can be given a precise definition in terms of the theory of real and complex finite dimensional algebras. We show that such systems can be regarded as systems over algebras (or rings) and that in this context the constraint of structural preservation is simply the usual requirement that operators (gain matrices, state and control matrices, etc.) are algebra homomorphisms. Thus all of the methodology that has been developed for algebraic system theory can be applied to develop a comprehensive theory of systems with structural symmetry.

In addition, a sizeable collection of mathematical results can be brought to bear on system theoretic questions arising from the somewhat diverse area of decentralized control. The underlying algebraic structure serves as a unifying concept for the many diverse applications.

\section{Three applications}

\subsection{The twin lift concept}

The moving of loads with helicopters is a reasonably routine problem in commercial and military applications as long as the loads are small. For the last thirty years, there has been a steady increase in the effective payload that can be manoeuvred. However, this increase in payload has been at the expense of larger and more expensive helicopters. The Sikorski CH-53E is a typical large helicopter and has an effective payload of approximately 40000 lbs. A significant increase in the payload can only be achieved by the construction of yet larger and more expensive aircraft. The problems involved in the design and maintenance of such large aircraft are well documented (Carter et al. 1979). The conclusion drawn is that there is an upper 
bound imposed on effective payload by physical and economic constraints. However, there does not appear to be an upper bound on the loads that can be moved by air. Thus the concept of attaching several helicopters to the same load seems to be one of some practical importance. The problem has been considered and flight tested in the case of two helicopters, often called 'twin lift'. In Carter et al. (1979) there is a brief report of a flight test that was performed by Sikorski Aircraft in the late 1960s. The main conclusions were that the concept was viable but carried the penalty of a high pilot workload and a lack of manoeuvrability.

The system involved can be schematically modelled as in Fig. 1. The helicopters are spread by a rigid bar and the attachment is by means of cables. The length of the cables is an important parameter. The effect of these and other parameters on performance is examined in Lewis and Martin (1983).

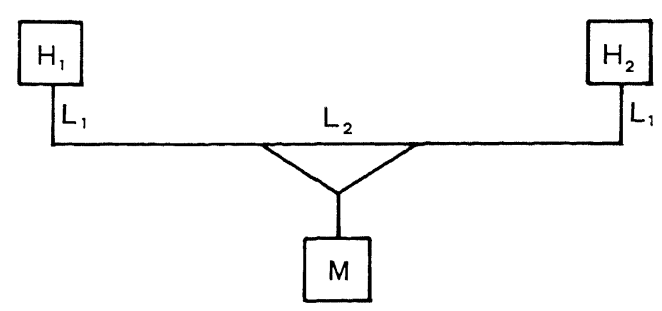

Figure 1.

If the mass is considered to be a point mass at the centre of the spreader bar and the helicopters are modelled with linear dynamics, the overall system model has the form

$$
\left(\begin{array}{l}
\dot{x} \\
\dot{y}
\end{array}\right)=\left(\begin{array}{cc}
A & H \\
-H & A
\end{array}\right)\left(\begin{array}{l}
x \\
y
\end{array}\right)+\left(\begin{array}{ll}
B & 0 \\
0 & B
\end{array}\right)\left(\begin{array}{l}
u_{\mathbf{1}} \\
u_{\mathbf{2}}
\end{array}\right)
$$

where the helicopter dynamics are modelled by

$$
\dot{x}=A x+B u
$$

and the matrix $H$ represents the coupling between the two systems. The matrix $H$ contains the effects of such parameters as $L_{1}$ and $L_{2}$. It is desirable that any control scheme should preserve the basic structure of eqn. (1). The local feedback of $x$ by $u_{1}$ and $y$ by $u_{2}$ should be given the same gain matrix $K$, both because of the desire to use all-purpose helicopters with identical avionics and for reasons of pilot training. Additional coupling between the two systems should be avoided, but if it cannot be, then it should preserve the physical dynamics already present. As we shall see and as is already documented (Hazewinkel and Martin 1983), the problem of stabilizing without additional coupling is very difficult even if possible. 


\subsection{Discretized partial differential equations}

Brockett and Willems (1974) considered the diffusion equation on a circle

$$
\frac{\partial x(t, \theta)}{\partial t}=\frac{\partial^{2} x(t, \theta)}{\partial \theta^{2}}+u(t, \theta), \quad 0 \leqslant \theta<2 \pi
$$

where $\theta$ is the angular coordinate and $0 \leqslant \theta \leqslant \pi$. By discretizing in the spatial domains, they arrived at an approximate ordinary differential equation

$$
\frac{d x_{i}(t)}{d t}=\frac{1}{a^{2}}\left[x_{i+1}(t)+x_{i-1}(t)-2 x_{i}(t)\right]+u_{i}(t), \quad i=1, \ldots, p
$$

where

$$
\left.\begin{array}{rl}
x_{i}(t) & =x\left(t, \frac{2 \pi}{p} i\right) \\
a & =\frac{2 \pi}{p}
\end{array}\right\}
$$

Because of the periodicity of the spatial domain, $k$ and $k+p$ are identified.

There is obviously special structure involved in eqn. (4), but it becomes much more obvious when the equation is written in state space form. Still following the development in Brocket and Willems (1974), we have the control system

$$
\frac{d}{d t}\left[\begin{array}{c}
x_{1}(t) \\
\vdots \\
\vdots \\
x_{p}(t)
\end{array}\right]=\frac{1}{a^{2}}\left[\begin{array}{ccccc}
-2 & 1 & 0 & \ldots & 1 \\
1 & -2 & 1 & \ldots & 0 \\
& & \vdots & & \\
1 & \ldots & 0 & 1 & -2
\end{array}\right]\left[\begin{array}{c}
x_{1}(t) \\
\vdots \\
\vdots \\
\vdots \\
x_{p}(t)
\end{array}\right]+\left[\begin{array}{c}
u_{1} \\
\vdots \\
\vdots \\
\vdots \\
u_{p}(t)
\end{array}\right]
$$

In their comprehensive treatment of such systems, Brockett and Willems (1974) noted that the system matrices had a great deal of algebraic structure and exploited the algebra to give a detailed analysis of such systems. They based their work on two points: that the matrices are circulant matrices; and that circulant matrices are related to a ring of polynomials $\mathbb{R}[z]$ where $z^{p}=1$.

We will show that their results can be viewed in much wider context and that most of their results hold. In particular, their ring is just a case of a group ring over a cyclic group.

\subsection{Command and control systems}

The problems of command, control, communication and intelligence have in the last few years received an increasing amount of attention from military bodies because of the realization of vulnerability of the existing structures to interference from hostile action. The control theorists have reacted with a major effort to bring to bear on this broad class of problems the resources of modern system analysis. The level of interest is reflected in the large number of papers available (for example, I.E.E.E. 1980). It should be realized that there are many types of problems encountered in command and control network and a wide variety of modelling and analysis techniques are of valid utility. 
Consider the problem of maintaining a formation of objects, be it ships, aircraft or a satellite communication network. For discussion, take a fleet of identical ships-perhaps destroyers protecting an aircraft carrier. There is limited communication between individual ships and they receive general commands as to heading and course from the carrier. For simplicity, assume that there are four destroyers arranged at the corners of a rectangle and communication is between adjacent ships as represented schematically in Fig. 2. If the dynamics of the ships are modelled by

$$
\dot{x}_{i}=A x_{i}+B u_{i}, \quad i=1, \ldots, 4
$$

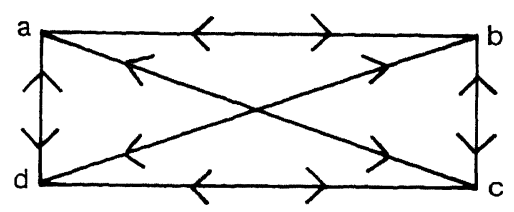

Figure 2.

the overall model is given by

$$
\left[\begin{array}{c}
\dot{x}_{1} \\
\dot{x}_{2} \\
\dot{x}_{3} \\
\dot{x}_{4}
\end{array}\right]=\left[\begin{array}{cccc}
A & H & H & H \\
H & A & H & H \\
H & H & A & H \\
H & H & H & A
\end{array}\right]\left[\begin{array}{l}
x_{1} \\
x_{2} \\
x_{3} \\
x_{4}
\end{array}\right]+\left[\begin{array}{cccc}
B & 0 & 0 & 0 \\
0 & B & 0 & 0 \\
0 & 0 & B & 0 \\
0 & 0 & 0 & B
\end{array}\right]\left[\begin{array}{l}
u_{1} \\
u_{2} \\
u_{3} \\
u_{4}
\end{array}\right]
$$

where the $H$ s represent the dynamic coupling induced by information exchange. The structure involved is central to the system and should be preserved. It may be necessary to modify the particular gains involved to achieve a stable and manoeuvrable formation but additional feedback loops would not be desirable. This linear model is such that it admits analysis of the effect of removing a node which is representative of the loss of a ship.

\section{Symmetry algebra}

In this section, we derive the basic definitions and theorems from algebra that are needed to study the systems described in $\S 2$. We will then associate with every class of systems an algebra which we will call the symmetry algebra. In Martin (1983) it was shown that systems could be associated with certain algebrae and in Hazewinkel and Martin (1983) it was shown that given a class, there is an implicitly associated algebra. Finally, we show that the system can be reduced to systems over the algebra.

\subsection{Algebrae and representations of algebrae}

We will work over a field $k$ that can usually be assumed to be either the field of real numbers $\mathbb{R}$ or as the field of complex numbers $\mathbb{C}$. Later we will 
have need of the non-comutative field of quaternions $\mathbb{H}$. Let $\mathbf{V}$ be a finite dimensional vector space with scalars from the field $k$. By an algebra over a field $k$ we mean a vector space $\mathbf{V}$ equipped with a multiplication, which we denote by juxtaposition that is distributive, associative and commutes with scalar multiplication. That is, if $\alpha \in k$ and $a, b \in \mathbf{V}$ then

$$
(\alpha a) b=a(\alpha b)=\alpha(a b)
$$

Standard examples are the complex numbers as a real algebra, the set of all $n \times n$ matrices, the algebra of polynomials in variables $x_{1}, \ldots, x_{n}$ and coefficients from $k$. Note that not every ring is an algebra : for example, the integers, while being a ring, do not admit a scalar multiplication by any field, and the real numbers are not an algebra over the complex numbers.

A module $\mathbf{M}$ over an algebra $\mathbf{A}$ is a vector space over $k$ and is closed under multiplication by elements of $\mathbf{A}$. It is easy to think of an $\mathbf{A}$-module as a vector space whose scalars are from $\mathbf{A}$. However, this can be misleading because many standard vector space results are not valid, for example the concepts of basis, dimension, inner products, etc. are not always definable. Let $\mathbf{M}_{1}$ and $\mathbf{M}_{2}$ be modules over $\mathbf{A}$. A module homomorphism between $\mathbf{M}_{\mathbf{1}}$ and $\mathbf{M}_{2}$ is a linear map from $\mathbf{M}_{1}$ to $\mathbf{M}_{2}$ (considered as vector spaces over $k$ ) that commutes with the multiplication by $\mathbf{A}$. Let $T$ be a linear map from $\mathbf{M}_{1}$ to $\mathbf{M}_{2}, v \in \mathbf{M}_{1}$ and $a \in \mathbf{A}$. Then $T$ is a homomorphism if and only if

$$
T(a v)=a(T v)
$$

Likewise, a homomorphism of two algebras $\mathbf{A}$ and $\mathbf{B}$ is a linear map of the underlying vector spaces that preserves the algebra multiplication.

A representation of an algebra $\mathbf{A}$ is an algebra homomorphism $\tau$ from $\mathbf{A}$ into the full matrix algebra $g l(\mathbf{V})$ for some vector space $\mathbf{V}$. Note that $\mathbf{V}$ can always be considered as a $g l(\mathbf{V})$ module and hence by the representation $\tau$ can be considered as an $\mathbf{A}$-module.

We often refer to the representation $(\tau, V)$ as the representation $V$ and denote $\tau(r) v$ as $r v$. A classical example of a representation in linear algebras comes from considering the representation of $\mathbb{R}[x]$, the ring of polynomials, in $g l(\mathbf{V})$. Let $\mathbf{A}$ be a fixed element of $g l(\mathbf{V})$ and define

$$
\tau(p(x)) v=p(A) v
$$

This representation was used to study a 'a single linear transformation' (Jacobson 1975).

We now recall some basic facts from representation theory. Let $V$ be a representation of an algebra $R$. A subspace $W \subseteq V$ is a subrepresentation if $w \in W$ implies that $r w \in W$ for all $r \in R$. A representation $V$ is irreducible if the only subrepresentations are 0 and $V$. An algebra $R$ is semisimple if for every representation $V$ and every subrepresentation $W$ there is a $W^{\prime}$ that is a subrepresentation and $W \oplus W^{\prime}=V$ as vector spaces. This has a practical interpretation in terms of matrices. If $\tau: R \rightarrow g l(V)$ is such that there exists a matrix $S \in G l(V)$ and $S^{-1} \tau(r) S$ is block upper triangular for all $r \in R$, then there is a $P \in G l(V)$, the group of invertible linear maps from $V$ to $V$, such that $P^{-1} \tau(r) P$ is block diagonal for all $r \in R$. The field $k$ of scalars is not important for the above. Standard examples of semisimple algebrae include 
$g l(V, \mathbb{R}), g l(V, \mathbb{C})$, the quaternions and the complex numbers as algebrae over $\mathbb{R}$ and the group algebra of any finite group (Serre 1977) over $\mathbb{R}$ or $\mathbb{C}$.

One of the main theorems of representation theory is Schur's lemma. We recall here three special cases. Let $\mathbf{V}$ be a representation of $R$ and let $\operatorname{End}_{R}(\mathbf{V})$ be the algebra of all $R$-homomorphisms of $\mathbf{V}$ into $\mathbf{V}$. The cases are as follows.

(1) Let $R$ be an algebra over $\mathbb{C}$ and $\mathbf{V}$ an irreducible complex representation of $R$. Then $\operatorname{End}_{R}(\mathbf{V})=\mathbb{C}$.

(2) Let $R$ be an algebra over $\mathbb{R}$ and $\mathbf{V}$ an irreducible real representation. Then $\operatorname{End}_{R}(\mathbf{V})=\mathbb{R}, \mathbb{C}$ or $\mathbb{H}$.

(3) If $\mathbf{V}$ and $\mathbf{M}$ are non-isomorphic irreducible representations of $R$, then $\operatorname{Hom}_{R}(\mathbf{V}, \mathbf{M})=0$ where $\operatorname{Hom}_{R}(\mathbf{V}, \mathbf{M})$ is the vector-space of $R$ homomorphisms from $\mathbf{V}$ to $\mathbf{M}$. (This is true for $\mathbb{R}$ and $\mathbb{C}$ ).

\subsection{Definition of symmetry algebra}

Let $g l(n, \mathbb{R})$ be the algebra of $n \times n$ matrices and let $\mathbf{C}$ be a class of systems of state dimension $n$ and input dimension $m$. Then the symmetry algebra of C is defined by

$$
\begin{aligned}
& R(\mathbf{C})=\{(S, T) \in g l(n, \mathbb{R}) \times g l(m, \mathbb{R}): \\
& \qquad S F=F S, \quad S G=G T, \quad \text { for all }(F, G) \in \mathbf{C}\}
\end{aligned}
$$

Usually $R(\mathbf{C})$ is uniquely determined by the projection into $g l(n, \mathbb{R})$. This happens if $\mathbf{C}$ contains a single system $(F, G)$ with $G$ having full rank for then $S G=G T$ determines $T$ uniquely as a function of $S$. This does not mean that $R(\mathbf{C})=\{S \in g l(n, \mathbb{R}): S F=F S\}$.

Consider the examples from $\S 2$. The first example was the twin lift helicopter. The class

$$
\mathbf{C}=\left\{\left(\left(\begin{array}{cc}
A & H \\
-H & A
\end{array}\right),\left(\begin{array}{cc}
B & 0 \\
0 & B
\end{array}\right)\right): A, H \text { and } B \text { as defined }\right\}
$$

The symmetry algebra is the set of $(S, T)$ such that

$$
S=\left(\begin{array}{cc}
\alpha I_{n} & \rho I_{n} \\
-\rho I_{n} & \alpha I_{n}
\end{array}\right), \quad T=\left(\begin{array}{cc}
\alpha I_{m} & \rho I_{m} \\
-\rho I_{m} & \alpha I_{m}
\end{array}\right)
$$

and hence $R(\mathbf{C})$ is a representation of $\mathbb{R}[i]$, the complex numbers as an algebra over $\mathbb{R}$.

The second example of Brockett and Willems (1974) has C as the class of systems with the state matrix as a circulant matrix and the input matrix the identity and hence $R(\mathbf{C})$ is a representation of $\mathbb{R}[z]$ with $z^{p}=1$. For this example, $\mathbb{R}[z]$ is also the group ring of $C_{p}$, the cyclic group of order $p$.

The third example has the set $\mathbf{C}$ described by eqn. (8) and since the model is symmetric under all permutations of the four ships the symmetry algebra is the group ring of the symmetric group on four letters, $S_{4}$. 
The three algebrae of the examples are quite diverse but an algebraist might see features shared by the three and for example, draw the conclusion that all symmetry algebrae are semisimple. The following theorem shows that no conclusions can be drawn about the general structure of symmetry algebrae.

\section{Theorem 1}

Every finite dimensional associative algebra with identity occurs as the symmetry algebra of some class of systems.

The proof of this theorem is contained in Hazewinkel and Martin (1983) and will not be repeated here. The proof is similar to the proof that for a 'wild 'quiver every algebra occurs as the endomorphism algebra (Hazewinkel 1976, Kleiner and Martin 1981). Thus the study of special classes of linear systems with structure makes non-trivial contact with the theory of the representation of algebrae.

\subsection{Dimension reduction}

Let $R \subseteq G l(n, \mathbb{R}) \times G l(n, \mathbb{R})$ and consider the class of systems with special structure $R$, state space $\mathbb{R}^{n}$ and input space $\mathbb{R}^{m}$, via the imbedding $R \subseteq M_{n}(\mathbb{R}) \times$ $I_{m}(\mathbb{R})$ they become (left) $R$-modules. Now let $\Sigma=(A, B) \in \mathbf{C}(R)$. Then $A S=S^{\prime} A, S B=B T$ for all $(S, T) \in R$ which precisely means that $A: R^{n} \rightarrow R^{n}$ and $B: R^{m} \rightarrow R^{n}$ are $R$-module homomorphisms. Thus we can consider $\Sigma=(A, B) \in \mathbf{C}(R)$ as a system over the ring $R$. Of course, there is no guarantee that the $R$-modules $\mathbb{R}^{n}$ and $\mathbb{R}^{m}$ will be free $R$-modules.

However, as we shall see, especially if the ring $R$ is semisimple, it may be advantageous to consider a system in $\mathbf{C}(R)$ as a system over $R$. In particular, if we are dealing with systems over $\mathbb{C}$ with special structure and $R$ is semisimple then the theory of systems with special structure $R$ is naturally equivalent to the theory of the usual linear systems over $\mathbb{C}$. If we are dealing with real systems and $R$ is semisimple, then the theory of systems with special structure $R$ reduces to the union of the theory of ordinary real systems, ordinary complex systems and linear systems over the (non-commutative) field of the quaternions.

\section{The theory of systems with semisimple symmetry algebra}

We recall from $\S 3.1$ that an algebra $R$ is semisimple if and only if every subrepresentation is complimented or in matrix terms if $R$ is algebra isomorphic to a direct sum of algebrae $R_{i}$ with $R_{i}$ a complete matrix algebra over some field. In this section, we first consider the simpler case when $R$ is a complex algebra and then the case that $R$ is a real algebra.

\subsection{Systems with complex semisimple symmetry algebra}

Let $\mathbf{C}$ be a class of complex systems with complex symmetry algebra $R \subset g l(n, \mathbb{C}) \times g l(m, \mathbb{C})$ and we assume that $R$ is semisimple. Let $(\boldsymbol{F}, G) \in \mathbf{C}$. We let the state space $\mathbb{C}^{n}$ and the input space $\mathbb{C}^{m}$ be $R$-modules by the embedding of $R$ into $g l(n, \mathbb{C}) \times g l(m, \mathbb{C})$. By the definition of the symmetry algebra and $\S 3.3$, we have that $F$ and $G$ are $R$-module homomorphisms. 
We write $\mathbb{C}^{n}$ and $\mathbb{C}^{m}$ as the direct sum of irreducible $R$-modules, i.e.

$$
\begin{aligned}
& \mathbb{C}^{n}=\overbrace{V_{1}+\ldots+V_{1}}^{n_{1}}+\overbrace{V_{2}+\ldots+V_{2}}^{n_{2}}+\ldots+\overbrace{V_{k}+\ldots+V_{k}}^{n_{k}} \\
& \mathbb{C}^{m}=\overbrace{W_{1}+\ldots+W_{1}}^{m_{1}}+\overbrace{W_{2}+\ldots+W_{2}}^{m_{2}}+\ldots+\overbrace{W_{p}+\ldots+W_{p}}^{m_{p}}
\end{aligned}
$$

Note that $G$ is an $R$-module homomorphism and hence $G: W_{1} \rightarrow \mathbb{C}^{n}$ and by Schur's lemma $W_{1}$ must be one of the $V_{i}$ s or $G \mid W_{1}=0$. Thus, if we assume, as is customary, that $G$ has full rank, then we assume without loss of generality that $W_{i}=V_{i}$ for $i=1, \ldots, p$. On the other hand, if $(F, G)$ is controllable then we must have that $p=k$ and we have then that

$$
\begin{aligned}
& \mathbb{C}^{n}=V_{1}^{n_{1}}+\ldots+V_{k}^{n_{k}} \\
& \mathbb{C}^{m}=V_{1}^{m_{1}}+\ldots+V_{k}^{m_{k}}
\end{aligned}
$$

and that $F$ and $G$ can be written as the direct sums

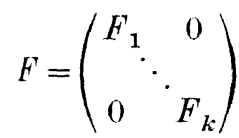

$$
\begin{aligned}
& G=\left(\begin{array}{ccc}
G_{1} & & 0 \\
& \ddots & \\
0 & & G_{k}
\end{array}\right)
\end{aligned}
$$

where $F_{i}$ is the restriction of $F$ to $V_{i}{ }^{n_{i}}$ and $G_{i}$ is the restriction of $G_{x}$ to $V_{i}{ }^{m_{i}}$.

Repeated applications of Schur's lemma to the summand $F_{i}$ gives that $F_{i}$ can be written as

$$
\left(\begin{array}{ccc}
f_{1,1} I_{d_{i}} & \cdots & f_{1 n_{i}} I_{d_{i}} \\
& \vdots & \\
f_{n_{i}, 1} I_{d_{i}} & \cdots & f_{n_{i}, n_{i}} I_{d_{i}}
\end{array}\right)
$$

where $d_{i}$ is the dimension of $V_{i}$ and $f_{j, k}$ is a complex scalar. Letting $F_{i}=\left(f_{j k}\right)$ we then have the form for

$$
F=\left(\begin{array}{ccc}
F_{1} \otimes I_{d_{1}} & & 0 \\
& \ddots & \\
0 & & F_{k} \otimes I_{d_{k}}
\end{array}\right)
$$

A similar result holds for $G$ to give

$$
G=\left(\begin{array}{ccc}
G_{1} \otimes I_{d_{1}} & & 0 \\
& \ddots & \\
0 & & G_{k} \otimes I_{d_{k}}
\end{array}\right)
$$

Thus, $F$ and $G$ can be reduced to the direct sum of ordinary complex linear systems by a change of basis in state space that preserves the symmetry algebra. All of the usual system theoretic questions concerning $(F, G)$ reduce to questions over lower dimensional complex systems. We have proven the following theorem. 


\section{Theorem 2}

Let $R$ be a semisimple subalgebra of $g l(n, \mathbb{C}) \times g l(m, \mathbb{C})$ and let $(F, G)$ be a system with symmetry algebra $R$. Let $\left(F_{1} G_{1}\right), \ldots,\left(F_{k}, G_{k}\right)$ be the subalgebrae constructed as above. Then the following conditions hold.

(i) $(F, G)$ is controllable if and only if each $\left(F_{i}, G_{i}\right)$ is controllable.

(ii) The characteristic polynomial of $F, C(F)$, is equal to

$$
C(F)=C\left(F_{1}\right)^{d_{1}} \ldots C\left(F_{k}\right)^{d_{k}}
$$

(iii) $(F, G)$ is stable if and only if $\left(F_{i}, G_{i}\right)$ is stable for each $i$.

The practical advantage of Theorem 2 is that it has reduced the theory of a class of structured systems to the theory of ordinary linear systems with complex coefficients. Thus, the question of what system theoretic operations can be performed within the class $\mathbf{C}$ is answered.

\subsection{Systems with real semisimple symmetry algebra}

Most physical systems are modelled by models having real coefficients. As we shall see, the theory of real systems is somewhat more complicated than the theory of complex systems. But in the case when the symmetry algebra is semisimple, much can be done.

As in $\S 4.2$, we consider $\mathbb{R}^{n}$ and $\mathbb{R}^{m}$ as $R$-modules and decompose them into the direct sum of irreducible $R$-modules. In this case, we get the following decomposition

$$
\left.\begin{array}{rl}
\mathbb{R}^{n}= & \overbrace{V_{1} \oplus \ldots \oplus V_{1} \oplus \ldots \oplus \overbrace{V_{k} \oplus \ldots \oplus V_{k}}^{\alpha_{1}}}^{\alpha_{k}} \\
& \overbrace{U_{1} \oplus \ldots \oplus U_{1} \oplus \ldots \oplus \overbrace{U_{p} \oplus \ldots \oplus U_{p}}^{\beta_{1}}}^{\beta_{p}} \\
& \overbrace{W_{1} \oplus \ldots \oplus W_{1} \oplus \ldots \oplus \overbrace{W_{s} \oplus \ldots \oplus W_{s}}^{\gamma_{1}}}^{\gamma_{s}}
\end{array}\right\}
$$

The $V_{i} \mathrm{~s}$ have the property, from Schur's lemma, that $\operatorname{End}_{R}\left(V_{i}\right)=\mathbb{R}$; the $U_{i} \mathrm{~s}$ the property that $\operatorname{End}_{R}\left(U_{i}\right)=\mathbb{C}$; and the $W_{i} \mathrm{~s}$ the property that $\operatorname{End}_{R}\left(W_{i}\right)=\mathbb{H}$. By repeating the tedious arguments of $\$ 4.2$, using schur's lemma repeatedly, we see that every system in $\mathbf{C}$ can be reduced to the direct sum of ordinary real systems, ordinary complex systems and 'ordinary' quaternion systems. Of course, it is not clear what an ordinary system with quaternion coefficients might look like. The theory of such systems is beyond the scope of this paper. We will assume in this paper that the quaternion components of $\mathbb{R}^{n}$ and $\mathbb{R}^{m}$ are not present. It seems to be not well understood in the mathematical literature how to impose conditions on the algebra $R$ to force this assumption. Thus the theory of real systems with semisimple structure algebra reduces to the theory of ordinary real systems and ordinary complex systems. The theory of quaternion systems is left to a later paper. 
If $\mathbb{R}^{n} \rightarrow \mathbb{R}^{m}$ is a homomorphism of $R$-modules, it preserves the special structure algebra $R$. It breaks up into a direct sum of feedback matrices corresponding to real and complex ordinary systems. Thus we have the following theorem.

\section{Theorem 3}

(i) $(F, G)$ is stabilizable by special structure preserving feedback if and only if all the real systems $\left(F_{i}, G_{i}\right), i=1, \ldots, k$ are stabilizable by real feedback and all the complex systems $\left(F_{k+i}, G_{k+i}\right), i=1, \ldots, p$ are stabilizable by complex feedback.

(ii) If $(F, G)$ is completely reachable, then the coefficients of its characteristic polynomial can be assigned arbitrarily by special structure preserving feedback subject to the sole condition that the characteristic polynomial must be of the form

$$
\begin{aligned}
p_{1}(\lambda)^{\alpha_{1}} \ldots p_{k}(\lambda)^{\alpha_{k}}\left(q_{1}(\lambda)^{\beta_{1}} \bar{q}_{1}(\lambda)\right)^{\beta_{p}} \ldots\left(q_{p}(\lambda) \bar{q}_{p}(\lambda)\right) & \\
& \text { degree }\left(p_{i}\right)=n_{i}, \quad \text { degree }\left(q_{i}\right)=n_{r+i}
\end{aligned}
$$

\section{Remark}

If $R=\mathbb{R}[Z /(n)]$ the irreducible real representations of $R$ (or equivalently $Z /(n))$ are of course very well known. They are of dimension 1 or 2 and are given by mapping the generator $g$ of $Z /(n)$ to an $n$th root of unity (interpreted as a rotation through an angle $2 \pi n^{-1}$ ). The corresponding decomposition of a circulant matrix readily follows and using the results above all the results of Brockett and Willems (1974) concerning block circulant systems readily follow.

Consider the structured system

$$
\left(\begin{array}{c}
\dot{x}_{1} \\
\dot{x}_{2}
\end{array}\right)=\left(\begin{array}{cc}
A & H \\
-H & A
\end{array}\right)\left(\begin{array}{l}
x_{1} \\
x_{1}
\end{array}\right)+\left(\begin{array}{cc}
B_{0} & B_{1} \\
-B_{1} & B_{0}
\end{array}\right)\left(\begin{array}{l}
u_{1} \\
u_{2}
\end{array}\right)
$$

with $A, H, B_{0}$ and $B_{1}$ real matrices. This system is irreducible over the reals and has structure algebra $\mathbb{R}[i]=\mathbb{C}$. Every irreducible component associated with the complex part of the algebra has the above form over the reals. Thus the theory of real structured systems with semisimple algebra reduces to ordinary real systems and real systems with the above structure.

Systems of the above form have occurred in various problems in aerospact technology (Sidar 1981). We see that their occurrence is not an accident because that form will occur whenever there is special structure with semisimple algebra. In the three examples presented originally, the structure algebra is semisimple in each case.

It is also worth noting that in a class of systems with semisimple structure algebra, the linear quadratic methodology is applicable. Let $P$ be the linear transform that decompose the state space into the form of eqn. (9) and $T$ the linear transform that decomposes the input space. Let $(A, B)$ be any system in C. Then $\left(P A P^{-1}, P B T\right)$ is the direct sum of complex systems 
and real systems. Each real system can be optimized by a cost criteria of the form

$$
J_{i}(u)=\int_{0}^{\infty} x^{\prime} Q_{i} x-u^{\prime} R_{i} u
$$

where $Q_{i}$ and $R_{i}$ are positive hermitian forms. This translates to the real representation as

$$
Q_{1}+i Q_{2} \mapsto\left(\begin{array}{cc}
Q_{1} & Q_{2} \\
-Q_{2} & Q_{1}
\end{array}\right)
$$

where $Q_{1}$ and $Q_{2}$ satisfy only the condition that $Q_{1}+i Q_{2}$ is positive hermitian and $R_{i}$ satisfies a similar condition. Thus the system $\left(P A P^{-1}, P B T\right)$ can be optimized by a cost function of the form

$$
J(u)=\int_{0}^{\infty} x^{\prime} Q x+u^{\prime} R u
$$

where $Q$ is of the form

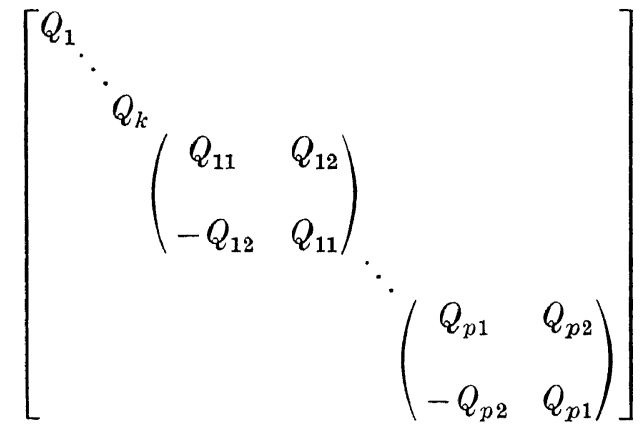

and the form of $R$ is similar.

Hence in $\mathbf{C}$ the system $(A, B)$ is optimized by

$$
J(u)=\int_{0}^{\infty} x^{\prime} P^{\prime} Q P x+u^{\prime} T^{\prime} R T u
$$

This emphasizes the fact that if the structure algebra is semisimple, then the standard algorithms may be used to effect modifications.

\section{Decentralized stabilization}

In this last section we present material that leads to an unsolved problem that is of some difficulty yet has some interesting consequences.

Consider the problem of stabilizing the pair of helicopters described in $\S 2.1$. As seen, this system can be considered as the complex system

$$
\dot{z}=(A+i H) z+B v
$$

The requirement that $u_{1}=K x_{1}$, and $u_{2}=K x_{2}$ then leads to requiring that the feedback control law for eqn. (10) be of the form

$$
u=K z
$$

In other words, we have a complex system which we wish to modify with real feedback. Note that this is dual (collequially) to output feedback. 
Here we have a system over a ring and we are asking for control using feedback that is restricted to a subring. The output feedback problem asks for control using a quotient of the state space.

Heymann's lemma applies to eqn. (10) to yield a new system

$$
\left(H+i H+B L, B u_{0}\right)
$$

where $L$ and $u_{0}$ are complex, which is controllable and single input. It is possible to show (Hazewinkel and Martin 1983) that there is a real $L$ and a real $u_{0}$ which also work. Thus we can assume that we have

$$
\dot{z}=(A+i H) z+b u
$$

which is controllable and single input. We can also assume without loss of generality that $(A, b)$ is in control canonical form, but we can make no assumptions about $H$. We now prove the following lemma.

\section{Lemma}

If $(A+i H, b)$ is in control canonical form then there exists a real feedback control law $k$ such that $A+i H+b k^{\prime}$ is stable, and furthermore given any real polynomial $p(\lambda)$ there is a feedback control law $k$ and a positive number $\epsilon$ such that the roots of the characteristic polynomial of $A+i H+b k^{\prime}$, are asymptotic to the roots of $p(\lambda / \epsilon)$.

\section{Proof}

Let

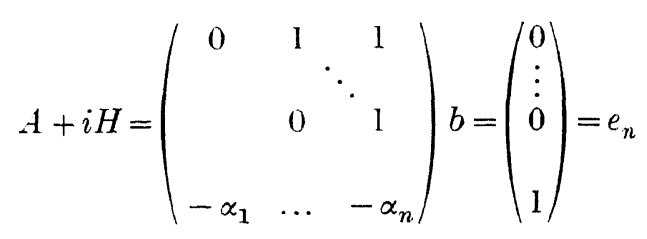

The $\alpha_{i} \mathrm{~s}$ are of course complex. Let the characteristic polynomial of $A+i H-b k^{\prime}$ be

$$
q(\lambda)=\lambda^{n}+\left(\alpha_{n}+k_{n}\right) \lambda^{n-1}+\left(\alpha_{n-1}+k_{n-1}\right) \lambda^{n-2}+\ldots+\left(\alpha_{1}+k_{1}\right)
$$

Let $p(\lambda)=\lambda^{n}+\beta_{n} \lambda^{n-1}+\ldots+\beta_{1}, \beta_{i} \in \mathbb{R}$. Now we define $k_{i}=\beta_{i} t^{n-i+1}$ and have

$$
q(\lambda, t)=\lambda^{n}+\left(\alpha_{n}+\beta_{n} t \lambda^{n-1}+\ldots+\left(\alpha_{1}+\beta_{1} t^{n}\right)\right.
$$

Factor out a factor of $t^{n}$ to yield

$$
q(\lambda, t)=t^{n}\left(\left(\frac{\lambda}{t}\right)^{n}+\left(\alpha n / t+\beta_{n}\right)(\lambda / t)^{n-1}+\ldots+\left(\alpha_{1} / t^{n}+\beta_{1}\right)\right)
$$

Thus for $t$ very large the roots of $q(\lambda, t)$ are approximately the roots of

$$
w^{n}+\beta_{n} w^{n-1}+\ldots+\beta_{1}
$$

where $w=\lambda / t$. Thus for $t$ very large and positive, the roots of $q(\lambda, t)$ are asymptotic to the roots of $p(\lambda / t)$. This finishes the proof of the lemma. 
But now suppose that $(A+i H, b)$ is not in canonical form. (We can still assume that $(A, b)$ is in canonical form). Then there is a complex matrix $T$ such that

and $T e_{n}=e_{n}$.

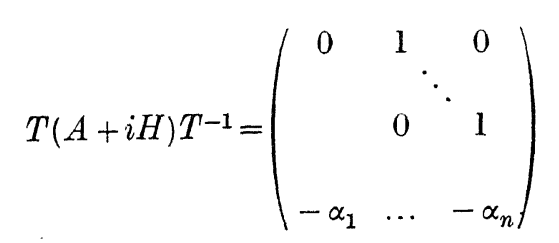

If we use real feedback on the transformed system then we have

$$
T(A+i H) T^{-1}+T b k^{\prime}
$$

has the required poles but we see that the original system has been modified by the complex feedback $k^{\prime} T^{-1}$. We are led then to the following question: given a system in canonical form, does there exist a feedback control law of the form $k^{\prime} T, T$ complex, $k$ real that stabilizes the system?

The answer is yes if $n=2$. However, the problem seems quite difficult if $n=3$. It should also be noted that in the case of helicopters the dimensions of interest are $n \geqslant 27$. From the Lemma we deduce that the above question is equivalent to the following more simple question.

Given $T$ complex, non-singular and $T e_{n}=e_{n}$, does there exist a real vector $k$ such that the polynomial

$$
p(\lambda)=\lambda^{n}+k^{\prime} T_{n} \lambda^{n-1}+\ldots+k^{\prime} T_{1}
$$

is stable, where $T_{i}$ is the $i$ th column of $T$.

\section{REFERENCES}

Allen Smith, G., 1981, personal communication.

Brockett, R., and WrLlems, J. L., 1974, Automatica, 10, 507.

Carter, E. S., Cooper, D. E., and Knapp, L. G., 1979, SAE Technical Paper 791087, Aerospace Meeting, Hyatt House, Los Angeles.

Hazewinkel, M., 1976, Proc. NASA-Ames Conf. on Geometric Control Theory (Brookline: Math Sci. Press).

Hazewinkel, M., and Martin, C., 1983, J. Pure Appl. Algebra (submitted).

I.E.E. Control Systems Society, 1980, Proc. Conf. Decision and Control.

JACobson, N., 1975, Lectures in Abstract Algebra II : Linear Algebra (New York: Springer-Verlag).

Kreiner, M., and Martin, C., 1981, Proc. MTNS Santa Monica, California.

Lewis, J., and Martin, C., 1983, A.I.A. Aerospace J. (submitted).

Mlartin, C., 1982, Int. J. Control, 35, 291.

Serre, J.-P., 1977, Linear Representations of Finite Groups (New York : SpringerVerlag).

Sidar, M., 1981, personal communication. 